\title{
Variabilidade espacial dos atributos físicos do solo e da produtividade em um Latossolo Bruno distrófico da região de Cascavel,PR ${ }^{1}$
}

1 Parte da dissertação de Mestrado apresentada pelo primeiro na UNIOESTE
${ }_{2}^{2}$ CCET/NIT/UNIOESTE. CEP 85814-110, Cascavel, PR. Fone: (45) 220-3134. E-mail: jerryaj@unioeste.br (Foto)
${ }^{3}$ CCET/UNIOESTE. Fone: (45) 223-4413. E-mail: godoy@unioeste.br.
${ }^{4}$ FEAGRI/UNICAMP. CP 6011, CEP 13083-970, Campinas, SP. Fone: (19) 3788-1060. E-mail: jansle@agr.unicamp.br

Protocolo 96 - 27/5/2003 - Aprovado em 16/2/2004

\begin{abstract}
Resumo: Através de técnicas de geoestatística foram confeccionados mapas de contorno, produzidos por interpolação através da krigagem ordinária representando, desta forma, a variabilidade espacial dos atributos físicos densidade do solo $\left[\mathrm{kg} \mathrm{dm}^{-3}\right]$, teor de água do solo $\left[\mathrm{g} \mathrm{g}^{-1}\right]$ e resistência mecânica a penetração [MPa] nas camadas de 0-10,10-20 e 20-30 cm de profundidade, além da produtividade de soja [t ha-1 ${ }^{-1}$. Os atributos do solo e a produtividade se derivaram de um plano de amostragem estratificada sistemática desalinhada, do ano agrícola 1998/99, subdividido em parcelas sem manejo localizado (SML) e parcelas com manejo localizado (CML). De maneira geral, os mapas de produtividade apresentaram um padrão de variabilidade semelhante quanto à distribuição na área não respondendo, assim, ao tratamento químico diferenciado aplicado nas parcelas CML; já os atributos físicos mostraram comportamento semelhante para os dois métodos de cultivo. Dentre os atributos físicos estudados, a resistência à penetração na camada $0-10 \mathrm{~cm}$ de profundidade foi a variável que melhor se correlacionou com a produtividade.
\end{abstract}

Palavras-chave: agricultura de precisão, geoestatística, soja

\section{Spatial variability of the physical attributes and the productivity in a distrophic Latosolo of Cascavel - PR, region}

\begin{abstract}
Through geostatistics techniques, contour maps, were produced by interpolation using ordinary kriging, representing the spatial variability of the physical attributes; soil density $\left[\mathrm{kg} \mathrm{dm}^{-3}\right]$, soil water $\left[\mathrm{g} \mathrm{g}^{-1}\right]$ and penetration resistance [MPa] in the 0-10, 10-20 and 20-30 $\mathrm{cm}$ of depth, besides the soy bean productivity [t ha $\left.{ }^{-1}\right]$. Soil attributes and yield data, derived from an unaligned stratified systematic sampling scheme, subdivided in portions with localized management (CML) and without localized management (SML) for the agricultural year 1998/99. The productivity maps in general presented a similar variability standard for the distribution in the non responding area as well as in the area with different chemical treatments applied in the CML plots. Where as the physical attributes presented a similar behavior for the two methods of management. Amongst the physical attributes studied, the penetration resistance in the $0-10 \mathrm{~cm}$ of depth was the variable which was best correlated with the productivity.
\end{abstract}

Key words: precision agriculture, geostatistics, physical attributes, productivity, soy bean

\section{INTRODUÇÃO}

O recente processo de globalização da economia vem impondo aos segmentos produtivos, dentre os quais o setor agrícola nacional, a obtenção de níveis de competitividade internacionais. Para atender a esse novo paradigma, novos conceitos, métodos e técnicas devem ser incorporados ao processo produtivo da agropecuária, envolvendo mudanças radicais de atitudes em relação ao que se pratica hoje. A agricultura brasileira necessita, assim, do desenvolvimento de tecnologias que possibilitem a competição de seus produtos no mercado mundial, através da ampliação da produtividade, o que é normalmente obtido pelo aumento do uso dos insumos agrícolas; porém, a falta de conhecimentos adequados sobre 
os diferentes ecossistemas e a conseqüente utilização de tecnologias inadequadas, têm contribuído para a perda de competitividade econômica do setor agrícola e para a degradação ambiental. Os problemas ambientais que podem surgir no setor agrícola envolvem a água, o ar, a qualidade dos alimentos e a depreciação de recursos naturais. De acordo com Rocha \& Lamparelli (1998) as aplicações convencionais de fertilizantes atualmente realizadas pelos agricultores, significam aplicações excessivas em determinadas áreas do campo e insuficientes em outras, em função da não uniformidade natural existente nos solos; desta forma, a adequação da utilização de insumos dentro das reais necessidades de cada tipo de solo e para cada tipo de cultura, é essencial para a promoção da sustentabilidade do setor, tanto a nível econômico como ambiental.

Considerando-se que a variabilidade existente deva ser incorporada aos procedimentos e tecnologias aplicados na agricultura, há alguns anos agricultores estão adotando um novo sistema de produção denominado, no Brasil, Agricultura de Precisão (AP). Seu principal conceito é aplicar no local correto, no momento adequado, as quantidades de insumos necessários à produção agrícola, para áreas cada vez menores e mais homogêneas, tanto quanto a tecnologia e os custos envolvidos o permitam (Manzatto et al., 1999).

A adoção desta nova tecnologia se justifica porque, atualmente, a grande maioria dos produtores agrícola considera uniforme o solo de cada área de cultivo e cada talhão pode ter consideráveis variações em seus atributos, tais como variabilidade espacial do tipo de solo, da produtividade, da declividade e da necessidade de nutrientes. Assim, a otimização das quantidades aplicadas de corretivos, fertilizantes, herbicidas, fungicidas, inseticidas e água irrigada, pode ajudar a maximizar os lucros e proteger o meio ambiente com a redução do uso indiscriminado e irracional de insumos e, para tanto, há necessidade de se conhecer os diversos fatores que influenciam a produtividade de uma cultura, em que uma das formas é o estudo da variabilidade espacial existente no solo, no intuito de minimizar análises laboratoriais e despesas com coleta de dados a campo.

Os parâmetros que influenciam a agricultura são variáveis em relação ao tempo e ao espaço. Dada a gama de fatores e parâmetros envolvidos, a agricultura de precisão requer um monitoramento contínuo, espacial e temporal, da área estudada, resultando em um grande volume de dados derivados de sensores ou de observações a campo, através de análises laboratoriais. Assim, cada vez mais o manuseio de ferramentas e técnicas como o Sistema de Posicionamento Global (GPS), Sensoriamento Remoto (SR) e o Sistema de Informação Geográfica (SIG) serão necessários.

Enquanto em AP os mapas de variabilidade espacial da produtividade são gerados a partir de um conjunto de sensores (ex: de fluxo de massa e de posicionamento espacial), permitindo uma cobertura de toda a área, os dados relativos às variáveis ligadas ao solo são baseados em processos de amostragem, havendo necessidade de se gerar uma superfície contínua, expressa em um mapa, por meio de um processo de interpolação digital. Para que se possa estudar esta variabilidade espacial, baseada em amostras, é conveniente utilizar-se técnicas como a geoestatística. A geoestatística permite estabelecer um modelo de semivariograma que melhor descreva a variabilidade espacial dos dados, o qual será utilizado no processo de interpolação digital pelo método da krigagem.

Entretanto, de acordo com Guimarães (2000) para que a geoestatística possa ser usada, há necessidade de que pelo menos uma das três hipóteses de estacionariedade de uma função aleatória $Z_{(\mathrm{xi})}$ seja satisfeita: a hipótese de estacionariedade de ordem 2 , a hipótese intrínseca e a hipótese de não tendência. Uma vez avaliadas as hipóteses envolvidas, Guimarães (1993), Vieira (1998) e Pannatier (1996) definiram o semivariograma clássico de Matheron como um método gráfico que expressa a estrutura espacial da semivariância $\gamma(\mathrm{h})$.

Por sua vez, Mingoti \& Silva (1995) comentam que o estimador clássico de Matheron é considerado não viciado para $\gamma(h)$ quando a função aleatória é um processo intrinsecamente estacionário, mas é pouco resistente e muito afetado por observações atípicas (outliers). Cressie \& Hawkins (1980) citados por Ribeiro Júnior (1995) comentam ainda que, sob normalidade, o estimador de Matheron é estável, mas tal fato não ocorre quando são simuladas distribuições de caudas pesadas, casos em que deve ser utilizado um estimador de Cressie \& Hawkins.

Segundo Ribeiro Junior (1995), a estimação da estrutura de dependência espacial do semivariograma é um passo crítico e trabalhoso, visto que requer paciência, várias tentativas $\mathrm{e}$ avaliações, uma vez que o modelo de variabilidade espacial escolhido será tomado como livre de erro e utilizado nas predições a serem feitas. Conforme Vieira (1998), em um semivariograma são estimados os parâmetros efeito pepita ("nugget effect"), patamar ("sill") e alcance ("range") representados, respectivamente, pelos símbolos " $\mathrm{C}_{0}$ ", " $\mathrm{C}_{0}+\mathrm{C}_{1}$ " $e$ "a". O valor de " $\mathrm{C}_{0}$ " revela a descontinuidade do semivariograma para distâncias menores que aquelas amostradas ou o próprio erro amostral. $\mathrm{O}$ alcance "a" é considerado a distância limite da dependência espacial, a partir da qual as amostras são consideradas independentes e, portanto, $(2)$ estatística clássica pode ser usada sem restrição.

No presente trabalho analisaram-se os resultados da segunda amostragem de solo (abril/1998) em relação à variabilidade espacial das propriedades físicas e densidade do solo; teor de água do solo e resistência mecânica a penetração nas camadas de 0-10, 10-20 e 20-30 cm de profundidade, assim como da produtividade da soja para o ano agrícola 98/99, em uma área experimental com parcelas sem manejo localizado (SML) e outras com manejo localizado (CML).

\section{MATERIAL E MÉTODOS}

Este estudo foi realizado em uma área de Latossolo Bruno Distrófico (EMBRAPA, 1999) pertencente ao Centro de Pesquisa Eloy Gomes da Cooperativa Central Agropecuária de Desenvolvimento Tecnológico e Econômico Ltda (COODETEC) situado em Cascavel, Paraná, em parceria com a Universidade Estadual do Oeste do Paraná (UNIOESTE) através do Núcleo de Inovações Tecnológicas (NIT). A área foi considerada plana, visto que as declividades foram menores que $0,19 \%$.

Em função dos resultados obtidos na primeira amostragem do solo, realizada em abril de 1998, cada parcela do experimento foi preparada da seguinte forma: as parcelas brancas (SML) 
receberam corretivo (calcário) e/ou fertilizantes em razão da média global obtida por todas as parcelas brancas e cada parcela cinza (CML), em função de sua individual necessidade, conforme esquema demonstrando na Figura 1. Após o tratamento diferenciado das parcelas, realizou-se o plantio da soja do ano agrícola 1998/99, em sistema de semeadura direta.



Figura 1. Esquema da amostragem desalinhada sistemática estratificada

Após a colheita desta soja, realizada em abril de 1999, foram demarcadas novamente as 256 parcelas da área experimental, em uma grade ("grid") de 7,20 x 7,20 m, com carreador de 2,40 m em uma das direções (Figura 1). Adotou-se uma bordadura de $1,10 \mathrm{~m}$ a fim de eliminar a influência do tratamento de uma parcela sobre outra, reduzindo cada parcela a 5,0 x $5,0 \mathrm{~m}\left(25 \mathrm{~m}^{2}\right)$ totalizando uma área útil cultivada de 1,33 ha. O objetivo desta coleta foi levantar as informações de solo para o ano de 1999. Por se tratar de parcelas pequenas, a colheita foi realizada com uma colhedora de parcelas de propriedade da COODETEC, especialmente produzida para colheita de experimentos.

Os locais de amostragem dentro de cada parcela experimental foram determinados de forma aleatória, seguindo-se o procedimento denominado amostragem desalinhada sistemática estratificada, demonstrado na Figura 1 (Wollenhaupt \& Wolkowski, 1994). Este procedimento combina os benefícios de uma grade ("grid") regular e uma amostragem aleatória. Maiores detalhes a respeito da metodologia empregada podem ser encontrados em Souza et al. (1998).

Os atributos físicos do solo avaliados para o ano de 1999 foram: densidade do solo (Dens.) $\left[\mathrm{kg} \mathrm{dm}^{-3}\right]$, teor de água do solo (Umid.) [\%] e resistência mecânica a penetração [MPa] nas camadas de 0-10 $\left(\mathrm{IC}_{0-10}\right), 10-20\left(\mathrm{IC}_{10-20}\right), 20-30 \mathrm{~cm}\left(\mathrm{IC}_{20-30}\right)$ de profundidade. Neste trabalho, estudou-se a variabilidade espacial desses atributos físicos e da produtividade da soja para as parcelas SML e CML.

A densidade do solo foi determinada pelo método do anel volumétrico (Khiel, 1979) com uma repetição por parcela e o teor de água do solo o foi pelo método gravimétrico padrão (EMBRAPA, 1979). A resistência mecânica a penetração foi definida por meio de um penetrógrafo (características prescritas pela ASAE S313.2/1982) com quatro repetições por parcela, realizadas de forma aleatória em cada parcela experimental, definindo um ponto médio de resistência mecânica a penetração nos cartões, para cada uma das camadas estudadas.
Inicialmente, realizou-se uma análise exploratória geral e uma exploratória espacial dos dados. A primeira não considera a posição geográfica de cada amostra coletada; assim, foram encontrados média, mediana, quartis, desvio padrão, coeficiente de variação, assimetria e curtose, através dos softwares estatísticos STATISTICA 5.0 e MINITAB 12.2. Os valores de assimetria e curtose encontrados para os dados, foram confrontados com os limites inferiores e superiores sugeridos por Jones (1969) para 128 dados a um nível de significância de $5 \%$; já na análise exploratória espacial dos dados, as posições de cada amostra são consideradas no estudo, caso em que foi construído o gráfico post-plot no software EXCEL, onde as amostras foram representadas no ponto em que foram coletadas (coordenadas) e cada parcela do experimento foi classificada segundo os seus quartis. Este gráfico mostra o comportamento espacial das realizações obtidas da variável em questão, na região estudada e a partir dela se pode verificar a existência de sub-regiões com comportamento semelhante na área em estudo.

Após esta análise preliminar, os dados foram mensurados sob a ótica da geoestatística, no intuito de se identificar à estrutura de variabilidade espacial. Para identificação da estrutura de dependência espacial entre as amostras, utilizouse o semivariograma clássico de Matheron, definido na Eq. 1, para os atributos que apresentaram normalidade. Aqueles atributos que não possuíam distribuição normal, foram modelados pelo semivariograma de Cressie \& Hawkins, definido na Eq. 2:

$$
\begin{gathered}
\gamma(h)=\frac{1}{2 N(h)} \sum_{i=1}^{N(h)}\left[z\left(x_{i}\right)-z\left(x_{i}+h\right)\right]^{2}, \\
\gamma(h)=\frac{1}{2} \frac{\left[N(h)^{-1} \sum_{N(h)} \sqrt{|Z(x i+h)-Z(x i)|}\right]^{4}}{0,457+\frac{0,494}{N(h)}}
\end{gathered}
$$

em que $N(h)$ : número de pares de valores medidos $Z_{(x i)}, Z_{(x i+h)}$, separados por um vetor de distância $h ; Z_{(x i)}=$ variável aleatória em estudo na i-ésima posição,

Basicamente, o processo consistiu em se ajustar um modelo teórico, esférico, exponencial, gaussiano, ao semivariograma experimental encontrado através do software VARIOWIN 2.2 (Pannatier, 1996) e IDRISI 3.2 (Eastman, 1999), respectivamente, para as Equações 1 e 2. O próximo passo foi estimar os valores em posições não amostradas no campo através da técnica de krigagem ordinária com o uso do software SURFER 6.0 (Golden Software, 1997) gerando, desta forma, os mapas de contorno (isolinhas) para as variáveis estudadas.

Para todas as variáveis em estudo foram produzidos mapas do comportamento espacial, subdividindo-os inicialmente em dez classes e tomando-se o cuidado de que os mapas para os dois métodos de cultivo (SML e CML) fossem representados segundo uma mesma escala de classificação. O menor e o maior valor da escala de cada mapa foram definidos tomando-se como 
base os menores e maiores valores encontrados nos dois mapas interpolados (ferramenta "grid info" do software Surfer 6.0); em seguida, esses mesmos mapas foram reclassificados objetivando-se assim, melhor caracterização prática da variabilidade encontrada. Para os parâmetros densidade do solo e resistência mecânica a penetração do solo, adotou-se a reclassificação sugerida, respectivamente, por Brady (1983) e Assis et al. (2001).

Uma avaliação quantitativa da variabilidade espacial também foi realizada; para tanto, utilizou-se o "coeficiente efeito pepita" $\left(\mathrm{E}_{\mathrm{O}}\right)$, como sendo a relação percentual entre o efeito pepita e o patamar, ou seja, $\mathrm{C}_{0} /\left(\mathrm{C}_{1}+\mathrm{C}_{0}\right)$. Quanto maior este coeficiente, menor a variabilidade espacial. $\mathrm{O}$ "coeficiente de efeito pepita" de até $25 \%$ do patamar, foi classificado como forte dependência espacial; entre 25 e $75 \%$, como moderado e, acima de $75 \%$, como fraca dependência espacial (Souza et al., 1999, Fietz et al., 2000).

\section{RESULTADOS E DISCUSSÃO}

Na Tabela 1 apresenta-se a análise exploratória geral dos valores encontrados para as variáveis produtividade da soja (Prod.) [ $\left.\mathrm{t} \mathrm{ha}^{-1}\right]$; densidade do solo (Dens.) $\left[\mathrm{kg} \mathrm{dm}^{-3}\right]$; teor de água do solo (Umid.) [ $\left.\mathrm{g} \mathrm{g}^{-1}\right]$; resistência mecânica a penetração [MPa] nas camadas de 0-10 $\left(\mathrm{IC}_{0-10}\right), 10-20\left(\mathrm{IC}_{10-20}\right), 20-30 \mathrm{~cm}\left(\mathrm{IC}_{20}\right.$ ${ }_{30}$ ) de profundidade para as parcelas sem manejo localizado (SML) e para as parcelas com manejo localizado (CML).

A hipótese de normalidade dos dados foi avaliada pelos testes de Anderson-Darling e/ou Shapiro-Wilk, a nível de 5\% de significância, conforme Tabela 1; além disto, foram apresentadas as medidas de forma, ou seja, os coeficientes de assimetria e curtose de cada variável. Os referidos valores foram comparados com os valores da tabela de Jones (1969) a qual apresenta intervalo de $95 \%$ de confiança para a curtose e assimetria, caracterizando a distribuição normal de probabilidades.

Para as parcelas SML, as variáveis Prod. e $\mathrm{IC}_{0-10}$ não apresentaram normalidade dos dados, mas as demais variáveis a apresentaram, a nível de 5\% de significância, cujo resultado corroborou com as medidas de forma, curtose e assimetria, avaliadas pelos intervalos de Jones (1969); já para as parcelas CML, nenhuma variável estudada indicou normalidade dos dados.

Para avaliação de pontos discrepantes (outliers) foram construídos boxplot de cada variável estudada; de forma geral, percebeu-se que as variáveis que não apresentaram normalidade tiveram, como característica, outliers unilaterais.

A análise exploratória espacial teve como objetivo analisar a existência de tendências direcionais nos dados, necessária ao emprego de métodos de geoestatística. Quando os dados não possuem tendência direcional, diz-se que eles são isotrópicos. A verificação de tal tendência foi avaliada pelo gráfico da observação versus as coordenadas, Norte-Sul (N$\mathrm{S})$, Leste-Oeste (L-O), Nordeste-Sudoeste (Ne-So) e SudesteNoroeste (Se-No) e pelo coeficiente de correlação linear de Pearson. Embora tenha havido correlações significativas entre algumas variáveis e suas respectivas coordenadas, estas foram relativamente fracas, o que não justificou nenhuma transformação dos dados nem a eliminação de tendências.

Outra ferramenta importante na avaliação da estacionariedade ou tendência direcional, é o gráfico post-plot, no qual cada parcela do experimento é classificada segundo os seus quartis; assim, os dados que pertençam ao mesmo intervalo quartílico são classificados e representados como iguais e a concentração de cores iguais no mapa pode indicar a existência de uma sub-região. Desta forma, se o mapa apresentar uma variação gradativa numa região ou direção, poderá estar indicando a existência de uma tendência ou falta de estacionariedade (Ribeiro Junior, 1995; Libardi, 1996; Silva, 2000).

As análises efetuadas através dos gráficos post-plot, scatterplot, média versus desvio padrão móvel e gráficos direcionais da variável resposta, corroboraram com a inexistência de tendências direcionais; nos semivariogramas construídos nas direções $0,45,90$ e $135^{\circ}$, verificou-se a inexistência de anisotropia; assim, estas variáveis parecem não mostrar problemas que afrontem as hipóteses necessárias ao emprego da geoestatística no estudo da variabilidade espacial dos dados. Assim, adotaram-se modelos isotrópicos, ou seja,

Tabela 1: Resultados estatísticos das variáveis em estudo para as parcelas sem manejo localizado (SML) e para as parcelas com manejo localizado (CML)

\begin{tabular}{|c|c|c|c|c|c|c|c|c|c|c|c|c|}
\hline \multirow{2}{*}{ Variável } & \multicolumn{2}{|c|}{ Prod.(t ha $\left.{ }^{-1}\right)$} & \multicolumn{2}{|c|}{ Dens. $\left(\mathrm{kg} \mathrm{dm}^{-3}\right)$} & \multicolumn{2}{|c|}{ Umid. $\left(\mathrm{g} \mathrm{g}^{-1}\right)$} & \multicolumn{2}{|c|}{$\mathrm{IC}_{0-10}(\mathrm{MPa})$} & \multicolumn{2}{|c|}{$\mathrm{IC}_{10-20}(\mathrm{MPa})$} & \multicolumn{2}{|c|}{$\mathrm{IC}_{20-30}(\mathrm{MPa})$} \\
\hline & SML & CML & SML & CML & SML & CML & SML & $\mathrm{CML}$ & SML & CML & SML & CML \\
\hline $\mathrm{N}^{o}$ de casos válidos & 128 & 127 & 128 & 128 & 128 & 128 & 128 & 128 & 128 & 128 & 128 & 128 \\
\hline Média & 1,96 & 1,94 & 1,20 & 1,20 & 0,32 & 0,32 & 3,35 & 3,56 & 2,61 & 2,59 & 2,45 & 2,41 \\
\hline Mínimo & 0,40 & 0,68 & 1,08 & 1,03 & 0,26 & 0,17 & 1,80 & 1,70 & 1,70 & 1,50 & 1,60 & 1,40 \\
\hline Primeiro Quartil & 1,78 & 1,74 & 1,16 & 1,16 & 0,30 & 0,31 & 2,90 & 2,90 & 2,40 & 2,40 & 2,30 & 2,30 \\
\hline Mediana & 1,98 & 2,05 & 1,19 & 1,20 & 0,32 & 0,32 & 3,20 & 3,20 & 2,60 & 2,50 & 2,50 & 2,50 \\
\hline Terceiro Quartil & 2,26 & 2,22 & 1,23 & 1,22 & 0,33 & 0,33 & 3,68 & 3,70 & 2,80 & 2,80 & 2,60 & 2,58 \\
\hline Máximo & 2,93 & 2,81 & 1,34 & 1,35 & 0,37 & 0,42 & 5,20 & 5,40 & 3,50 & 3,60 & 3,10 & 3,50 \\
\hline Desvio Padrão & 0,45 & 0,44 & 0,05 & 0,05 & 1,90 & 2,69 & 0,67 & 0,73 & 0,32 & 0,32 & 0,28 & 0,27 \\
\hline $\mathrm{CV}(\%)$ & 22,96 & 22,68 & 4,17 & 4,17 & 5,99 & 8,53 & 19,88 & 21,47 & 12,18 & 12,44 & 11,39 & 11,19 \\
\hline Assimetria & $-0,80$ & $-0,81$ & $0,39^{*}$ & $0,11^{*}$ & $-0,13^{*}$ & $-1,62$ & 0,74 & 0,88 & $0,39 *$ & $0,20^{*}$ & $-0,28 *$ & $-0,26^{*}$ \\
\hline Curtose & 1,26 & $0,54 *$ & $-0,31^{*}$ & 1,36 & $0,04^{*}$ & 11,91 & $0,32 *$ & $0,42^{*}$ & $0,33^{*}$ & 1,48 & $0,42 *$ & 3,43 \\
\hline Normalidade (p-valor) & 0,01 & 0,01 & $0,10^{*}$ & 0,05 & $0,10^{*}$ & 0,01 & 0,01 & 0,01 & $0,10^{*}$ & 0,01 & $0,10^{*}$ & 0,01 \\
\hline
\end{tabular}

* Os dados apresentaram normalidade pelos testes de Anderson-Darling e/ou Shapiro-Wilk a nível de 5\% de significância 
assumiu-se que o padrão da estrutura espacial é o mesmo em todas as direções. A próxima etapa foi estudar os estimadores das estruturas de dependência espacial através de semivariogramas de cada variável. Para as variáveis que apresentaram distribuição normal dos dados, adotou-se o estimador clássico de Matheron e, para as demais variáveis, o estimador robusto de Cressie \& Hawkins.

Para as parcelas SML e CML, os parâmetros e modelos ajustados ao semivariograma experimental de cada variável em estudo, são apresentados, respectivamente, nas Tabelas 2A e B.

Para as parcelas SML (Tabela 2A) e de acordo com a classificação de $\mathrm{E}_{0}$ dada por Souza et al. (1999) e Fietz et al. (2000), nenhuma variável apresentou forte variabilidade espacial; somente a variável "Dens." mostrou fraca variabilidade espacial e as demais variáveis, apresentaram moderada variabilidade espacial dos dados; já para as parcelas CML (Tabela 2B) mais uma vez nenhuma variável exprimiu forte variabilidade espacial. A variável IC $_{20-30}$ foi a única a apresentar fraca variabilidade espacial e as demais variáveis, mais uma vez, apresentaram moderada variabilidade espacial dos dados.

Numa avaliação comparativa da dependência espacial dos dados para as parcelas SML e CML percebeu-se que, com exceção da variável produtividade, todas apresentaram menor dependência espacial para as parcelas CML. Uma explicação plausível para este comportamento diferenciado da variável produtividade nas parcelas CML, pode ter sido a aplicação diferenciada de insumos realizada nessas parcelas, fazendo com que a dependência espacial aumentasse em função de uma uniformização da produtividade para as referidas parcelas. Por outro lado, não se encontrou uma explicação razoável que evidenciasse uma dependência espacial menor das demais variáveis para as parcelas CML, visto que não foram realizadas práticas de manejo diferenciadas na área, que pudessem afetar os atributos físicos.

Tabela 2. Resultados da análise geoestatística para as parcelas

\begin{tabular}{llllrl}
\hline Variável & Modelo & Co & \multicolumn{1}{c}{$\mathrm{C}_{1}$} & $\mathrm{a}(\mathrm{m})$ & $\mathrm{E}_{\mathrm{O}}$ \\
\hline \multicolumn{4}{l}{ A. Sem manejo localizado (SML) } \\
Prod.** & Gaussiano & 0,161 & 0,089 & 68,1 & 65 \\
Dens.* & Gaussiano & 0,002 & 0,0005 & 126,0 & 82 \\
Umid.* $^{*}$ & Esférico & 1,98 & 2,45 & 140,0 & 45 \\
$\mathrm{IC}_{0-10} * *$ & Gaussiano & 0,313 & 0,292 & 85,7 & 52 \\
$\mathrm{IC}_{10-20} *$ & Exponencial & 0,053 & 0,053 & 68,8 & 50 \\
$\mathrm{IC}_{20-30} *$ & Esférico & 0,056 & 0,024 & 44,8 & 70 \\
\hline
\end{tabular}

B. Com manejo localizado (CML)

$\begin{array}{llllrl}\text { Prod.** } & \text { Exponencial } & 0,1485 & 0,187 & 149,2 & 44 \\ \text { Dens.** } & \text { Exponencial } & 0,0013 & 0,0015 & 60,0 & 46 \\ \text { Umid.** } & \text { Esférico } & 5,19 & 3,85 & 54,4 & 57 \\ \mathrm{IC}_{0-10} * * & \text { Gaussiano } & 0,349 & 0,204 & 51,4 & 63 \\ \mathrm{IC}_{10-20} * * & \text { Exponencial } & 0,064 & 0,039 & 26,0 & 62 \\ \mathrm{IC}_{20-30} * * & \text { Gaussiano } & 0,060 & 0,014 & 23,7 & 81\end{array}$

$\left(^{*}\right)$ Estimador de Matheron; (**) Estimador de Cressie \& Hawkins

Co - Efeito Pepita; a - Alcance; Eo - Coeficiente Efeito Pepita;
Com base nessas informações, foi possível a geração de um mapa representando uma superfície contínua para cada variável em estudo, caracterizando o comportamento de cada variável no campo. Em média, para todas as variáveis em estudo não houve diferenças significativas entre as parcelas SML e CML, conforme mostram as estatísticas descritivas da Tabela 1; entretanto, para que fosse possível a comparação espacial entre as parcelas SML e CML, foram confeccionados e apresentados os mapas temáticos lado a lado, como pode ser visualizado da Figura 2.

A Figura 2A mostra o comportamento espacial da produtividade da soja para os dois métodos de cultivo. De forma geral, o padrão de variabilidade (tendência) é semelhante quanto à distribuição na área, embora os mapas sejam um pouco diferentes. Percebe-se, para os dois casos, que as menores produtividades estão localizadas na parte inferior direita e as maiores na parte inferior esquerda.

A região oeste do Paraná é caracterizada pelo cultivo maciço da cultura de soja e o município de Cascavel, onde o experimento foi instalado, assume posição de destaque neste panorama, caracterizado por apresentar elevadas produtividades de soja, variando entre 2,5 e 3,7 tha-1 para os anos que não apresentam baixos índices pluviométricos no verão, porém, para o ano de 1999 obtiveram-se produtividades médias de 1,96 e 1,94 tha ${ }^{-1}$, respectivamente, para as parcelas SML e CML, produtividades estas abaixo da esperada para a região; a justificativa para este fato foi uma estiagem logo após o plantio, fazendo com que um número menor de plantas emergisse, além da ocorrência de uma estiagem prolongada na época em que a cultura estava na fase de preenchimento de grãos afetando, desta forma, a produtividade da área. A Figura $2 \mathrm{~A}$ mostra que em aproximadamente $50 \%$ da área foram obtidas produtividades variando de 1,95 a 2,15 $\mathrm{tha}^{-1}$, para os dois métodos de cultivo.

$\mathrm{Na}$ Figura 2B tem-se o comportamento espacial da densidade do solo para os dois métodos de cultivo; de forma geral, o comportamento desta variável parece diferir de um método para outro.

De acordo com Brady (1983), a densidade de solos argilosos pode variar desde 1,00 até $1,60 \mathrm{~kg} \mathrm{dm}^{-3}$, dependendo das suas condições; assim, utilizando-se este critério, reclassificou-se a variável densidade do solo, conforme Figura 2C; depois desta reclassificação, verificou-se que os mapas são muito semelhantes e que a densidade do solo na área está variando de 1,10 a $1,30 \mathrm{~kg} \mathrm{dm}^{-3}$. Dentro deste intervalo aproximadamente $50 \%$ da área possuem densidades variando de 1,10 a $1,20 \mathrm{~kg}$ $\mathrm{dm}^{-3}$ e o restante de 1,20 a $1,30 \mathrm{~kg} \mathrm{dm}^{-3}$.

Cintra \& Mielniczuck (1983) citados por Guimarães (2000) discorreram, em seu trabalho, de que a densidade do solo acima de $1,30 \mathrm{~kg} \mathrm{dm}^{-3}$ ocasionou uma redução de $50 \%$ no comprimento de raízes da soja. O comprimento das raízes da soja é responsável pela absorção da água e dos nutrientes e, sendo assim, é importante que esses valores não sejam ultrapassados a fim de não comprometerem a produtividade da cultura. Para este caso, a densidade média do solo para os dois métodos de cultivo, foi de $1,20 \mathrm{~kg} \mathrm{dm}^{-3}$.

A Figura 2D mostra o comportamento espacial do teor de água no solo para os dois métodos de cultivo; de forma geral, 
A. Produtividade $-\mathrm{t} \mathrm{ha}^{-1}$
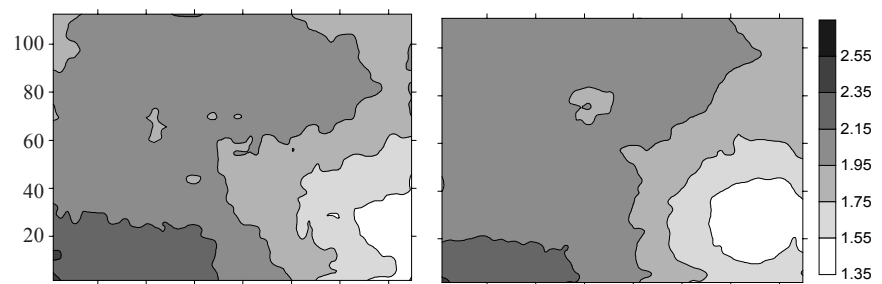

B. Desidade do solo - $\mathrm{kg} \mathrm{dm}^{-3}$
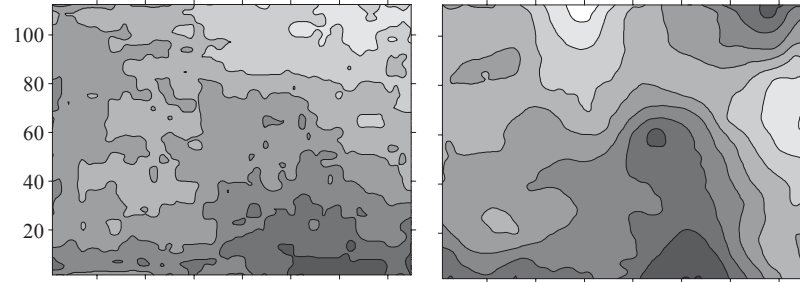

C. Desidade do solo $-\mathrm{kg} \mathrm{dm}^{-3}-\mathrm{RC}^{*}$
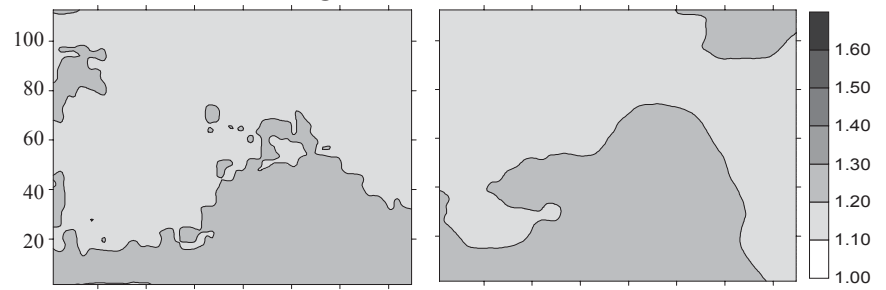

D. Teor de água no solo - $\mathrm{g} \mathrm{g}^{-1}$
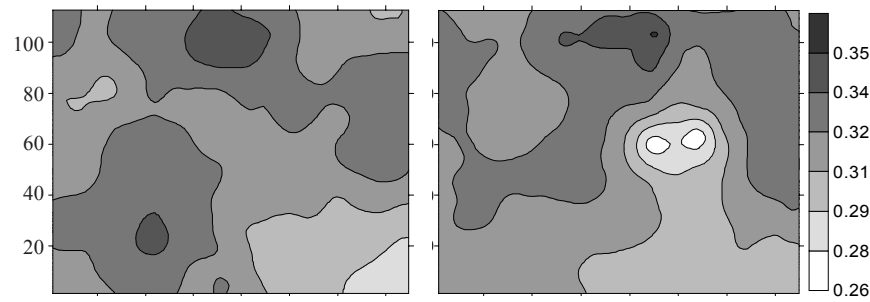

E. Resistência mecânica a penetração $(\mathrm{MPa})$ camada $0-10 \mathrm{~cm}$


F. Resistência mecânica a penetração (MPa) camada 0-10 cm- RC*

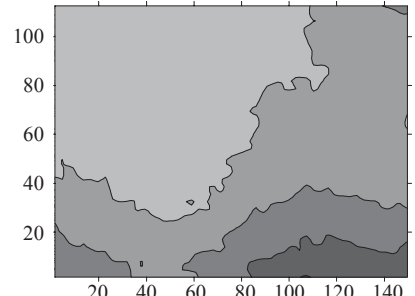

Sem manejo localizado

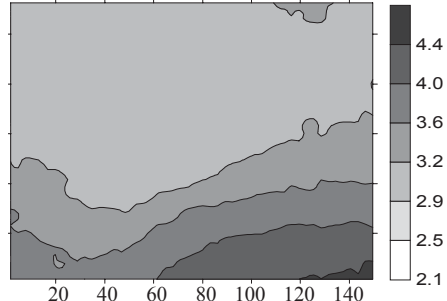

Com manejo localizado
G. Resistência mecânica a penetração (MPa) camada 10-20 cm
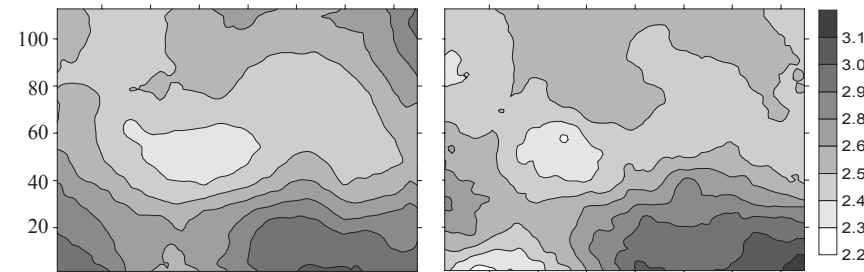

H. Resistência mecânica a penetração $(\mathrm{MPa})$ camada $10-20 \mathrm{~cm}-\mathrm{RC}^{*}$
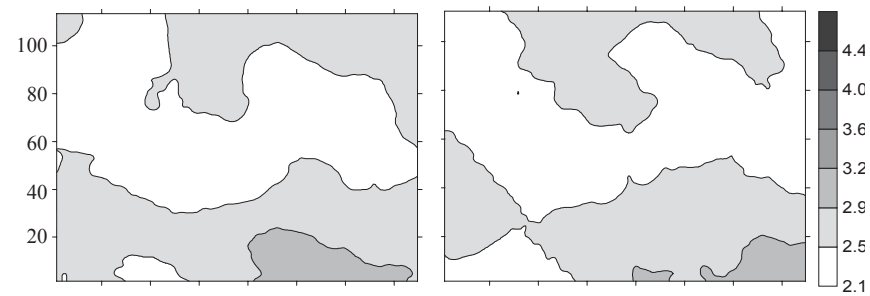

I. Resistência mecânica a penetração (MPa) camada $10-20 \mathrm{~cm}-\mathrm{RC}^{* *}$
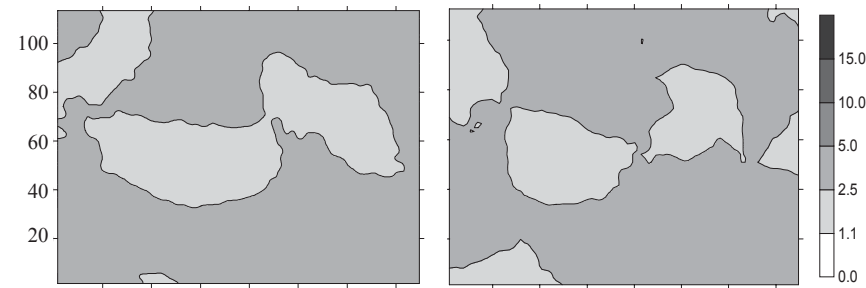

J. Resistência mecânica a penetração (MPa) camada 20-30 cm


K. Resistência mecânica a penetração( $(\mathrm{MPa})$ camada 20-30 cm-RC*

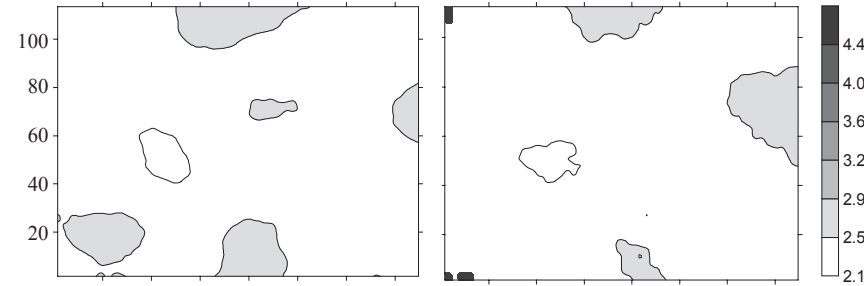

J. Resistência mecânica a penetração(MPa) camada 20-30 cm- RC**

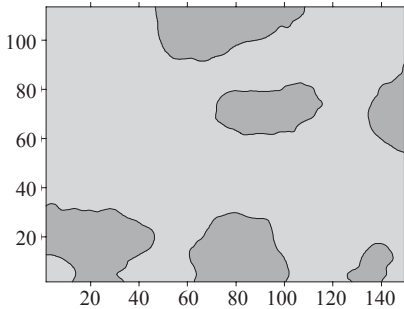

Sem manejo localizado



Com manejo localizado

RC*- Reclassificado; $\mathrm{RC}^{* *}$ - Reclassificado de acordo com Caranarache (1990) citado por Assis et al. (2001)

Figura 2. Mapas de diferentes atribultos estudados sob o metodo de cultivo sem manejo localizado e com manejo localizado

o padrão de variabilidade (tendência) é semelhante quanto à distribuição na área para os métodos em estudo, embora tenha havido maior variabilidade para as parcelas SML. Percebe-se, para os dois casos, que existe uma pequena "mancha" na área com teores de água acima de 0,34 e uma outra área um pouco maior na parte inferior direita, com teores de água variando de
0,28 a 0,31 , porém a maior parte da área em estudo teve o teor de água do solo variando entre 0,31 e 0,34.

A Figura 2E evidencia o comportamento espacial da resistência mecânica a penetração na camada de $0-10 \mathrm{~cm}$ de profundidade, para os dois métodos de cultivo. Para esta camada, a variável teve variação de 2,9 a 4,4 MPa; mais uma 
vez, visualiza-se um padrão de variabilidade (tendência) semelhante quanto à distribuição desta variável na área, para os métodos em estudo.

Para a reclassificação das três camadas em estudo, ou seja, $\mathrm{IC}_{0-10}, \mathrm{IC}_{10-20}$ e $\mathrm{IC}_{20-30}$, tomou-se como base a menor e a maior resistência mecânica a penetração encontrada para as três situações, variando de 2,1 a 4,4 MPa. Obtiveram-se, desta forma, sete classificações para esses mapas temáticos, como pode ser visto nas Figuras 2F, G e H; uma segunda reclassificação (Tabela 3) foi realizada conforme o que sugere Canarache (1990) citado por Assis et al. (2001) e pode ser visualizada nos mapas das Figuras 2I, J, K e L.

Tabela 3. Limites de classes de resistência de solos à penetração e graus de limitação ao crescimento das raízes

\begin{tabular}{lcl}
\hline Classes & Limites (MPa) & Limites ao crescimento das raízes \\
\hline Muito baixa & $<1,1$ & Sem limitação \\
Baixa & $1,1-2,5$ & Pouca limitação \\
Média & $2,6-5,0$ & Algumas limitações \\
Alta & $5,1-10,0$ & Sérias limitações \\
Muito Alta & $10,1-15,0$ & Raízes praticamente não crescem \\
Extremamente Alta & $>15,0$ & Raízes não crescem \\
\hline
\end{tabular}

Fonte: Adaptada de Canarache (1990) citado por Assis et al. (2001)

Para os primeiros $10 \mathrm{~cm}$ de profundidade visualiza-se na Figura $2 \mathrm{~F}$ que, de forma geral, o comportamento na área para os dois métodos de cultivo é semelhante. Praticamente, a única variação significativa ocorreu na parte superior direita do mapa SML; entretanto, a maioria da área em estudo mostrou valores variando de 2,9 a 3,2 MPa. A área mais compactada encontrase na parte inferior direita dos mapas, região esta próxima de uma estrada que dá acesso à área e a outras fazendas vizinhas à área experimental; assim, por se tratar de uma área mais sujeita ao tráfego de veículos, máquinas, implementos e pessoas, possivelmente tenha sido este o fator responsável pela maior compactação do solo. A princípio, este resultado parece correlacionar-se com a produtividade encontrada no campo, pois para este mesmo local se obteve uma produtividade menor nos dois métodos de cultivo. Esta variável não teve seus valores reclassificados, segundo a Tabela 3, em função dos valores encontrados estarem todos dentro da mesma faixa de classificação, ou seja, entre 2,6 a 5,0 MPa representando, segundo o autor, "algumas limitações ao crescimento das raízes".

O comportamento espacial da resistência mecânica a penetração na camada de $10-20 \mathrm{~cm}$ de profundidade para os dois métodos de cultivo, pode ser visualizado na Figura 2G. Para esta camada, obteve-se uma variação de 2,2 a 3,1 MPa evidenciando uma amplitude de variação bem menor que no caso anterior corroborando, assim, com o que já havia sido detectado através do coeficiente de variação. O comportamento espacial dos mapas é muito semelhante em toda a área, o que é evidenciado com a reclassificação (Figura $\mathrm{H}$ ), na qual se visualizam apenas três níveis de classificação nos mapas. A exemplo do que havia ocorrido na camada de $0-10 \mathrm{~cm}$, a área mais compactada se encontra na parte inferior direita dos mapas, o que se esperava, pelos motivos já apontados.
Pela Figura 2I visualiza-se que grande parte da área para os dois métodos de cultivo, se encontra na faixa de classificação entre 2,6 e 5,0 MPa representando, segundo o autor, "algumas limitações ao crescimento das raízes". Visualiza-se também, nestes mapas, uma segunda faixa de classificação variando de 1,1 a 2,5 MPa que representa conforme Canarache (1990) citado por Assis et al. (2001) "pouca limitação ao crescimento das raízes".

Finalmente, o comportamento espacial da resistência mecânica a penetração na camada de $20-30 \mathrm{~cm}$ de profundidade para os dois métodos de cultivo, pode ser visualizado na Figura 2J. Para esta camada, a variável mostrou variação entre 2,1 e 2,8 MPa com amplitude de variação ainda menor que as encontradas nos casos anteriores confirmando, mais uma vez, o que havia sido detectado na análise descritiva dos dados, através do coeficiente de variação; novamente, o comportamento espacial se assemelha entre os dois mapas, sendo evidenciado com a reclassificação (Figura $2 \mathrm{~K}$ ), na qual são encontradas apenas duas classificações no mapa proposto. Através dos mapas, percebe-se que aproximadamente $90 \%$ da área possuem seus níveis de compactação expressos entre 2,1 e 2,5 MPa na camada de 20-30 cm e o restante entre 2,5 e 2,0 MPa.

$\mathrm{Na}$ Figura 2L visualizar-se, para a camada de $20-30 \mathrm{~cm}$ de profundidade, que grande parte da área para os dois métodos de cultivo se encontra na faixa de classificação de 1,1 e 2,5 MPa que representa, segundo Canarache (1990) citado por Assis et al. (2001) "pouca limitação ao crescimento das raízes". O restante da área se encontra classificado entre 2,5 e 5,0 MPa, representando "algumas limitações ao crescimento das raízes".

Assis et al. (2001) relata que a compactação do solo pode influenciar a germinação, a brotação, a emergência das plantas, o crescimento radicular e, praticamente, todas as fases do desenvolvimento da cultura. Pelo estudo realizado percebeuse que a camada de $0-10 \mathrm{~cm}$ de profundidade foi a que apresentou a camada mais compacta decorrente, possivelmente, do uso intensivo do solo.

Autores como Canarache (1990) e Sene et al. (1985) citados por Assis et al. (2001) sugerem que solos argilosos começam a restringir o pleno desenvolvimento das raízes das plantas para valores acima de 2,5 MPa; assim, seguindo a sugestão dos autores, verifica-se a possibilidade da resistência mecânica a compactação do solo, principalmente nas duas primeiras camadas, ter sido uma das variáveis responsáveis por uma produtividade menor, correlação esta que já havia sido verificada quando da análise da camada de $0-10 \mathrm{~cm}$ de profundidade; outra variável que pode ter influenciado a produtividade, talvez seja o teor de água do solo durante o ciclo da cultura, uma vez que se teve um ano com períodos prolongados de estiagem.

\section{CONCLUSÕES}

1. Os mapas de produtividade apresentaram, de forma geral, um padrão de variabilidade semelhante quanto à distribuição na área não respondendo, desta forma ao tratamento químico diferenciado.

2. Um dos motivos para esta semelhança de produtividades foi, sem dúvida, a estiagem em duas fases da cultura, logo 
após o plantio e na época de enchimento de grãos, que fez com que a produtividade fosse abaixo da esperada, para a região.

3. Os atributos físicos densidade do solo, teor de água do solo e resistência mecânica a penetração nas camadas de 0 -10, 10-20 e 20-30 cm de profundidade, apresentaram comportamento semelhante para os dois métodos de manejo, o que se esperava, visto que não se realizou nenhum tipo de trato cultural que pudesse alterar as propriedades físicas do solo.

4. Dentre os atributos físicos estudados, a resistência mecânica a penetração na camada $0-10 \mathrm{~cm}$ de profundidade, foi a variável que melhor se correlacionou com a produtividade, pois nos locais onde ocorreram as menores produtividades se obteve maior índice de compactação e vice-versa.

\section{LITERATURA CITADA}

Assis, R.L.; Mion, R.L.; Ruiz, E.R.S.; Grossi, C.H.; Nery, M.S.; Lanças, K.P.; Antuniassi, U.R. Mapas de densidade e resistência mecânica à penetração do solo em diferentes profundidades no sistema de plantio direto em Botucatu In: Congresso Brasileiro de Ciência do Solo, 28, Londrina, PR, 2001. Anais... Londrina: SBCS, 2001. p.27-30.

Brady, N.C. Natureza e propriedades dos solos. 6 ed. São Paulo: Freitas Bastos, 1983, 623p.

Eastman, J.R. IDRISI32 - Worcester: Clark University, 1999. The Idrisi Project.

EMBRAPA - Serviço Nacional de Levantamento e Conservação de Solos. Manual de métodos de análise de solo. Rio de Janeiro:SNLCS, 1979. sp.

EMBRAPA - Serviço Nacional de Levantamento e Conservação de Solos. Sistema Brasileiro de classificação de solos. Brasília: Embrapa Produção de Informação, 1999, 412p.

Fietz, C.R.; Fabricio, A.C.; Salton, J.C. Mapa de aplicação localizada de calcário de uma área experimental. In: Balastreire, L.A. O estado-da-arte da agricultura de precisão no Brasil, Piracicaba. 2000. p.165-169,

Golden Software, INC. Surfer for Windows-User guide. Golden: Golden Software, Colorado, 1997.340p.

Guimarães, E.C. Variabilidade espacial da umidade e da densidade do solo em um Latossolo Roxo. Campinas: UNICAMP, 1993, 138p. Dissertação Mestrado

Guimarães, E.C. Variabilidade espacial de atributos de um Latossolo Vermelho Escuro textura argilosa da região do cerrado, submetido ao plantio direto e ao plantio convencional. Campinas: UNICAMP, 2000, 90p. Tese Doutorado.
Jones, T.A. Skewness and kurtosis as criteria of normality in observed frequency distribuitions. Journal Sedimentary Petrology, Colorado, p.1622-1627, 1969.

Khiel, E.J. Manual de edafologia: relações solo-planta. São Paulo: Ceres, 1979. 262p.

Libardi, P.L.; Manfron, P.A.; Moraes, S.O.; Toun, R.L. Variabilidade da umidade gravimétrica de um solo hidromórfico. Revista Brasileira de Ciência do Solo. Campinas, v.20, p.1-12, 1996.

Manzatto, C.V.; Bhering, S.B.; Simões, M. Agricultura de precisão: propostas e ações da Embrapa solos, 1999. http// www.cnps.embrapa.br/search/pesqs/proj01

Mingoti, S.A.; Silva, A.F. Um exemplo de aplicação de técnicas de geoestatística. Revista da Escola de Minas, Ouro Preto, v.49, p.96-100, 1995.

Pannatier, Y. Variowin 2.2: Software for spatial data analysis in 2D. New York: Springer, 1996. 96p.

Ribeiro Junior, P.J. Métodos geoestatísticos no estudo da variabilidade espacial de parâmetros do solo. Piracicaba: ESALQ 1995, 99p. Dissertação Mestrado

Rocha, J.V.; Lamparelli, R.A.C. Geoprocessamento. In: Silva, F.M. Mecanização e agricultura de precisão. Poços de Caldas: UFV, 1998, cap.1, p.1-30.

Silva, E.A.A. Aplicação de um estimador robusto na análise de variabilidade espacial de um Latossolo Roxo. Cascavel: UNIOESTE, 2000, 109p. Dissertação Mestrado.

Souza, E.G.; Johann, J.A.; Rocha, J.V.; Ribeiro, S.R.A.; Silva, M.S.; Uribe-Opazo, M.A.; Molin, J.P.; Oliveira, E.F.; Nóbrega, L.H.P. Variabilidade espacial dos atributos químicos do solo em um Latossolo Roxo distrófico na região de Cascavel, PR. Engenharia Agrícola, Jaboticabal, v.8, n.3, p.80-92, 1999.

Souza, E.G.; Ribeiro, S.R.A.; Uribe-Opazo, M.A.U.; Vilas Boas, M.; Silva, M.S.; Johann, J.A.; Molin, J.P.; Nóbrega, L.H.P.; Oliveira, E.F.; Carraro, I.A. Metodologia para análise da variabilidade espacial dos atributos do solo e da produtividade em uma área piloto de agricultura de precisão. In: Congresso Brasileiro de Engenharia Agrícola, 27, Poços de Caldas, Anais... Poços de Caldas: SBEA, v.3, 1998, p.109-111.

Vieira, S.R. Geoestatística aplicada à agricultura de precisão. In: GIS Brasil'98, Curitiba, PR, 1998, 53 p.

Wollenhaupt, N.C.; Wolkowski, R.P. Grid soil sampling. Better Crops with Plant Food, Norcross, v.78, n.4, p.6-9, 1994. 